\title{
Study of Human Serum Albumin-SPIONs Loaded PLGA Nanoparticles for Protein Delivery
}

\author{
Sri Vidawati, Silvia Barbosa, Pablo Taboada, Eva Villar, Antonio Topete, Victor Mosquera \\ Grupo de F'isica de Coloides y Pol'ımeros, Departamento de F'isica de la Materia Condensada, Universidad de Santiago de \\ Compostela, Santiago de Compostela, Spain \\ Email: pablo.taboada@usc.es
}

How to cite this paper: Vidawati, S., Barbosa, S., Taboada, P., Villar, E., Topete, A. and Mosquera, V. (2018) Study of Human Serum Albumin-SPIONs Loaded PLGA Nanoparticles for Protein Delivery. Advances in Biological Chemistry, 8, 91-100. https://doi.org/10.4236/abc.2018.85008

Received: August 16, 2018

Accepted: October 21, 2018

Published: October 24, 2018

Copyright (c) 2018 by authors and Scientific Research Publishing Inc. This work is licensed under the Creative Commons Attribution International License (CC BY 4.0).

http://creativecommons.org/licenses/by/4.0/

\begin{abstract}
The Biodegradable nanoparticles from poly(lactic-co-glycolic acid) (PLGA) have been extensively investigated for sustained and targeted/localized delivery of different agents. Many parameters are required in the synthesis of a biodegradable polymeric nanoparticle. We report the synthesis of human serum albumin (HSA)-superparamagnetic iron oxide nanoparticles (SPIONs) loaded PLGA nanoparticles. All nanoparticles were characterized using a TEM image, UV-Vis spectroscopy measurements, Zeta Potential, and PPMS for magnetizations. This study described and investigated the interesting phenomenon in the synthesis development of HSA-SPIONs loaded PLGA nanoparticles. The result showed that the stability of HSA-SPIONs loaded PLGA nanoparticles for potential applications such as in protein delivery.
\end{abstract}

\section{Keywords}

Nanoparticles, PLGA, SPIONs, HSA

\section{Introduction}

Nanotechnology is now used for various applications in agriculture [1], electronics [2], forensic science [3], space [4], medical therapeutics [5] [6] [7], etc. Nanotechnology has developed to such an extent that it has become possible to fabricate, characterize and specially tailor the functional properties of nanoparticles for biomedical applications and diagnostics [8] [9] [10]. A number of different polymers, both synthetic and natural, have been utilized in formulating biodegradable nanoparticles [11]. Biodegradable nanocarriers such as lipid or polymer based nanoparticles can be designed to improve the efficacy and reduce 
the toxic side effects of drugs. Nanocarriers can provide a crucial advantage to various drugs and therapeutic biological molecules such as nucleic acids and proteins by improving their efficacy and reducing potential toxic and side effects. Biodegradable nanoparticles offer possibilities to protect therapeutic agents against degradation, to control their release, to overcome biological barriers and to target specific sites of action [12] [13] [14] [15].

Biodegradable polymers based on PLGA are fully biocompatible and therefore among the most common materials used for encapsulating therapeutic agents. PLGA based nanoparticulate system is one of the most successfully used biodegradable polymers because PLGA-based nanoparticles can be used for the protection of the therapeutic agents from degradation, increase their stability, possibility of sustained release, possibility to target nanoparticles to specific organs or cells, and can be used for controlled delivery of therapeutics with improved pharmacokinetic and pharmaco dynamic profile. Another major advantage of PLGA over other polymers is that PLGA is approved by the United States Food and Drug Administration (US FDA) and European Medicines Agency (EMA) to be used in drug delivery systems in humans for parenteral administration, leading PLGA-based nanoparticles in a good position for clinical trials. Numerous antigens as proteins, peptides, lipopeptides, cell lysates, have been successfully formulated in PLGA nanoparticles [16] [17] [18] [19].

The concept of drug delivery using magnetic nanoparticles greatly benefit from the fact that nanotechnology has developed to a stage that it makes possible not only to produce magnetic nanoparticles in a very narrow size distribution range with superparamagnetic properties but also to engineer particle surfaces to provide site specific delivery of drugs. Magnetic properties were used first in biology and medicine for the magnetic separation of biological products and magnetic guidance of particle systems for site-specific drug delivery. The size, charge, and surface chemistry of magnetic particles could strongly influence their biodistribution. Another important point is that the magnetic properties depend strongly on the size of the magnetic particles. Very promising nanoparticles for these applications are SPIONs based on a core consisting of iron oxides that can be targeted through external magnets. SPIONs are coated with biocompatible materials and can be functionalized with drugs, proteins or plasmids. Based on their unique mesoscopic physical, chemical, thermal, and mechanical properties, SPIONs offer a high potential for several biomedical applications [20] [21]. One important advantage of the magnetic nanoparticle is their superparamagnetism that enables their stability and dispersion upon the removal of the magnetic field as no residual magnetic force exists between the particles.

Albumin, a versatile protein carrier for drug delivery, has been shown to be nontoxic, non-immunogenic, biocompatible and biodegradable. Nanoparticles made of albumin offer several specific advantages: they are biodegradable, easy to prepare and reproducible. Due to the high protein binding of various drugs, the matrix of albumin nanoparticles can be used for effective incorporation of these compounds [22]. Albumin nanoparticles have a bright future in the con- 
trolled delivery of therapeutic agents. Albumin nanoparticles showed a high drug loading capacity in combination with biodegradability, biocompatibility. A better understanding of the mechanisms of action of these vehicles will provide a basis for their further optimization, thus opening more exciting opportunities in the area of drug delivery [23].

HSA is an attractive macromolecular carrier and widely used to prepare nanospheres and nanocapsules. Among various available agents, HSA seems to be a promising molecule for the surface modification of polymeric PLGA nanoparticles. HSA is known to be the most abundant native protein in the human body which has various advantages, including ready availability, biodegradability, and low toxicity and immunogenicity [24]. HSA-based nanoparticles can be well tolerated without any serious side effects, which it is supported by clinical studies with registered HSA-based particle formulations. HSA has been shown to be biodegradable, nontoxic, easy to purify, and soluble in water, allowing ease of delivery by injection and thus an ideal candidate for nanoparticle preparation. The incorporation of suitable drugs in nanoparticles has been shown to protect pharmacologically active substances from degradation during storage as well as from early degradation/inactivation after injection [24] [25]. HSA covalently immobilized on modified magnetic nanoparticles so that it is significant for its magnetic applications in various bioprocesses, biomedical devices, and biomedicine [26]. The nanosystems for biomedicine consist of one or more magnetic cores and biological or synthetic molecules which serve as a basis for polyfunctional coatings on the magnetic nanoparticles surface. Proteins are promising materials for the creation of coatings on magnetic nanoparticles due to their biocompatibility, an ability to protect magnetic cores from the influence of biological liquids and prevent the agglomeration of magnetic nanoparticles in dispersion, their possible functional activity as therapeutic products.

One of the most frequently employed technique for the encapsulation of proteins into PLGA nanoparticles presents some challenges as instability problems [27]. The aim of this study described and investigated the interesting phenomenon in the development of encapsulated PLGA nanoparticles for protein delivery. Protein + SPIONs loaded PLGA nanoparticles have attracted their potential applications as protein carriers. To evaluate their potential as a protein carrier, HSA was used as a model protein for this study. We use encapsulated PLGA nanoparticles, which uses the combination of SPIONs and HSA. We report that the formation of these nanoparticles by characterizing their physicochemical properties, such as morphology, size, and surface chemistry can impact the biodistribution and pharmacokinetics of drugs by modifying interactions with the biological environment.

\section{Materials and Methods}

\subsection{Materials}

PLGA of 38 - $54 \mathrm{kDa}$ with 50:50 lactide-glycolide ratio, Pluronic $\mathrm{F}_{127}, \mathrm{FeCl}_{2}$, $\mathrm{FeCl}_{3}$, and Human Serum Albumin (HSA), were obtained from Sigma-Aldrich 
(St. Louis, MO, USA). Oleic acid with 90\% purity was obtained from Alfa Aesar (Karlshrue, Germany). All other chemicals and solvents were obtained from Sigma-Aldrich. Pure water of Milli-Q quality was used in all preparations.

\subsection{Synthesis of SPIONs}

Oleic acid-stabilized $\mathrm{Fe}_{3} \mathrm{O}_{4}$ SPIONs were synthesized by a co-precipitation method. Briefly, aqueous solutions of $0.1 \mathrm{M}^{\circ} \mathrm{FeCl}_{3}(30 \mathrm{~mL})$ and $\mathrm{FeCl}_{2}(15 \mathrm{~mL})$ prepared with $\mathrm{N}_{2}$ purged-water were mixed; then, $3 \mathrm{~mL}$ of $5 \mathrm{M}$ solution of ammonia was added in small aliquots of $0.6 \mathrm{~mL}$ while stirring. A black precipitate formed indicating the formation of SPIONs. After $20 \mathrm{~min}$ of stirring under the $\mathrm{N}_{2}$ atmosphere, $56.4 \mathrm{mg}$ of oleic acid was added to the SPIONs and the temperature was raised to $80^{\circ} \mathrm{C}$ and kept for $30 \mathrm{~min}$ while stirring to evaporate the ammonia. The magnetic nanoparticle was washed twice by centrifugation at $9000 \mathrm{rpm}$ for $20 \mathrm{~min}$ to remove excess of oleic acid, the supernatant was discarded and the precipitate was lyophilized and stored at $4^{\circ} \mathrm{C}$.

\subsection{Synthesis of HSA-SPIONs Loaded PLGA Nanoparticles}

The preparation polymeric encapsulated PLGA nanoparticles, containing the combination of SPIONs and HSA were prepared using the multiple emulsion solvent evaporation methods. In a typical preparation, PLGA $(25 \mathrm{mg})$ was dissolved in a sealed vial containing Dichloromethane (1 ml), HSA was dissolved in pure water $(100 \mu \mathrm{L})$ by ultrasonic $10 \mathrm{~min}$, and SPIONs dispersed in Dichloromethane by sonication with a probe-type sonicator $(20 \mathrm{kHz}$, Bandelin Sonopuls, Bandelin GmbH, Berlin, Germany) at some parameters of time and power in an ice bath. The combinations variation parameters of time and power of sonication were accurately very important in producing very high quality of polymeric PLGA nanoparticles with the combinations of SPIONs and HSA for protein delivery application. This study shows that the difference in the power and time of sonication parameters are very small and could yield significant and very different result from each synthesis HSA-SPIONs-PLGA nanoparticles.

Then, this organic solution was added drop wise with a syringe pump (0.166 $\mathrm{mL} / \mathrm{min})$ to an aqueous solution $\left(50 \mathrm{~mL}\right.$ ) containing Pluronic $\mathrm{F}_{127}$ (typically 1 wt $\%$ if not otherwise stated) while stirring at $10^{\circ} \mathrm{C}$. After sonication with power $100 \mathrm{~W}$ for 15 minutes of this experiment to homogenize the resulting dispersion, the organic solvent was completely evaporated under mechanical stirring overnight, the dispersion subsequently centrifuged twice at $9000 \mathrm{rpm}$ for $20 \mathrm{~min}$ and $20^{\circ} \mathrm{C}$. Subsequently, the supernatant was removed and the final precipitate was kept in the freezer.

\subsection{Characterization of Nanoparticles}

For this study, all of the nanoparticles were characterized using a TEM image, UV-Vis spectroscopy measurements, Zeta Potential, and PPMS for magnetizations.

Transmission electron microscopy (TEM) is the most powerful technique 
utilized in determining the particle size and morphology of nanoparticles. Samples were prepared for analysis by evaporating a drop of the nanoparticles dispersion on a carbon-coated copper grid without staining (TEM). TEM images of nanoparticles were obtained with a Philips CM-12 (Philips, Netherlands) microscope operating at $120 \mathrm{kV}$. HR-TEM images and selected area electron diffraction (SAED) patterns were obtained with a transmission electron microscope (Carl-Zeiss Libra 200 FE-EFTEM, Germany) operating at $200 \mathrm{kV}$.

UV-Vis measurements were used to determine structural, morphological particularities closely related to the SPIONs and HSA presence inside the polymeric PLGA nanoparticles. UV-VIS measurement also explained Human Serum Albumin (HSA) released. UV-V is spectroscopy measurements were performed in a CARY 100 Bio UV-Visible (Agilent Technologies, Santa Clara, USA) spectrophotometer.

Nanoparticles zeta potentials were obtained by triplicate with a Zetasizer Nano ZS (Malvern, UK), using disposable folded capillary cells. Each experiment was repeated at least three times. On the other hand, the magnetization of this experiment was measured using PPMS for magnetization. As a function of the applied magnetic field from $5 \mathrm{~K}$ until $300 \mathrm{~K}$. Magnetic susceptibility measurements were carried out with a SQUID magnetometer (Quantum Design MPMS5, San Diego, CA, USA).

\section{Result and Discussion}

\subsection{SPIONs}

SPIONs with appropriate surface chemistry have been widely used experimentally for numerous applications such as magnetic resonance imaging contrast enhancement, tissue repair, detoxification of biological fluids, hyperthermia, drug delivery and in cell separation, etc. SPIONs are small synthetic $\mathrm{Fe}_{2} \mathrm{O}_{3}$ or $\mathrm{Fe}_{3} \mathrm{O}_{4}$ particles with a core size of $<20 \mathrm{~nm}$ with an organic or inorganic coating. If the crystal size is small enough the thermal between the behavior of the small magnetic moment of a single paramagnetic atom and that of the much larger magnetic moment of a nanosized magnetic particle arises from the coupling of many atomic spins [11]. The quantum size effects and the large surface area of the magnetic nanoparticles dramatically change some of the magnetic properties and exhibit super paramagnetic phenomena and quantum tunnelling of magnetization, because each particle can be considered as a single magnetic domain [28].

In this study, oleic acid-stabilized SPIONs were obtained by a co-precipitation method. The TEM image of the size and morphology of SPIONs was shown in Figure 1. The TEM characterization of SPIONs was performed utilizing sized particle with a mean diameter of SPIONs around $5-20 \mathrm{~nm}$. The SPIONs (Figure 1) are apparently uniform and stable in the size and shape. The magnetic microspheres of SPIONs (with a diameter size of $5-20 \mathrm{~nm}$ ) in biocompatible, non-toxic (FDA approved) and biodegradable polymeric microspheres, such as 


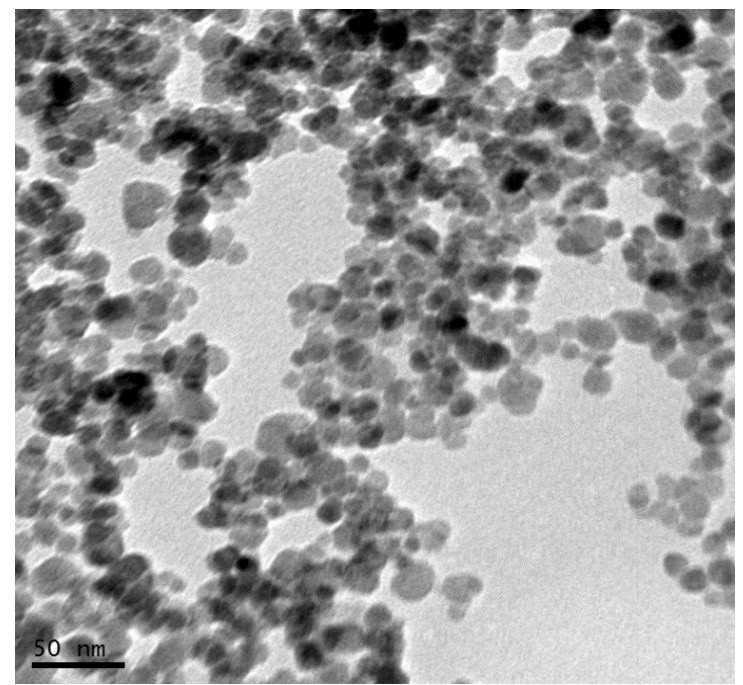

Figure 1. TEM image of superparamagnetic iron oxide nanoparticles (SPIONs).

polymeric PLGA nanoparticles are recognized as a promising drug carrier. The major advantage of magnetic microcarriers as compared with nanocarriers is their lower burst effect. The homogeneity of SPIONs is a distinguished feature of the biomedical application.

\subsection{Physicochemical Properties of HSA-SPIONs Loaded PLGA Nanoparticles}

Many studies that have been studied further about PLGA nanoparticles combinations with SPIONs and vaccines, or proteins, or nucleic acids, or amongst others [16]. The emulsification by sonication method allowed the preparation of spherical drug-loaded systems of biodegradable PLGA nano-carriers containing SPIONs and HSA. Preparation variables, such as the intensity, power, and time of sonication, are shown to be important parametric factors in the formation of PLGA nanoparticles for protein delivery. In this study, we informed the interesting phenomenon in the synthesis of biodegradable PLGA nanoparticles for protein delivery using the combination of SPIONs and HSA with the possibility of accurately processing the parameters, especially the variation parameter of time, the power of sonication and the concentration of each solution.

A double-emulsion method to produce PLGA nanoparticles with encapsulated SPIONs that uses oleic acid-coated magnetite nanoparticles dispersed in the oil phase has been developed emulsification is one of the key factors in the process of preparing nanoparticles because an insufficient dispersion of phase results in large particles with the widesize distribution. SPIONs were evenly distributed throughout the PLGA nanoparticles, both with respect to individual particles as well as with respect to many particles. The SPIONs appeared to uniformly assembled and distributed (Figure 1). Sonication with a probe-type sonicator $(20 \mathrm{kHz}$, Bandelin Sonopuls, Bandelin GmbH, Berlin-Germany) was used. Sonication mostly affects reaction rates, yields, and influenced in polymeric 
PLGA nanoparticles for protein delivery. The results of this section obtained that the processing parameter of sonication intensity played very important roles in the morphological and properties of HSA-SPIONs loaded PLGA nanoparticles.

Size and morphology of HSA-SPIONs loaded PLGA nanoparticles from this experiment were acquired by TEM. TEM image was used to obtain essential information on primary size and morphology of nanoparticles. TEM is an important technique that it unique capability of probing the internal structure of individual nanoparticles.

Figure 2 shows the TEM image of HSA-SPIONs loaded PLGA nanoparticles with sonication parameter of power $20 \mathrm{~W}$ for 12 minutes. In Figure 3, we used $0.1 \mathrm{~g} / \mathrm{ml}$ HSA-SPIONs loaded PLGA nanoparticles. TEM images in Figure 2 looked in the sphere nanocapsule and little bit aggregate in the nanocapsule. UV-Vis measurement informed HSA around $10 \%$ in the polymeric nanocapsule PLGA, and HSA releases around 5.5\% for 120 hours (see Figure 3).

In this study, HSA-SPIONs loaded PLGA nanoparticles have zeta potential around $-10.15 \mathrm{mV}$. The magnetization of this experiment was measured using PPMS. As a function of the applied magnetic field from $5 \mathrm{~K}$ until $300 \mathrm{~K}$, the result of magnetization of HSA-SPIONs loaded PLGA nanoparticles are shown in Figure 4. The nanoconstructs shown a typical ferromagnetic hysteresis loop such as the stability indicator of magnetic nanoparticles.

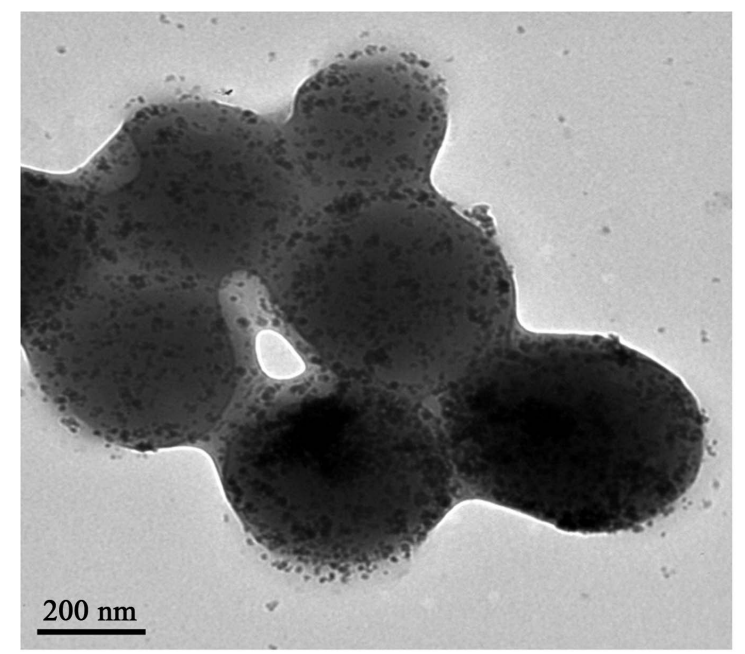

Figure 2. TEM images of the synthesis of HSA-SPIONs loaded PLGA nanoparticles.

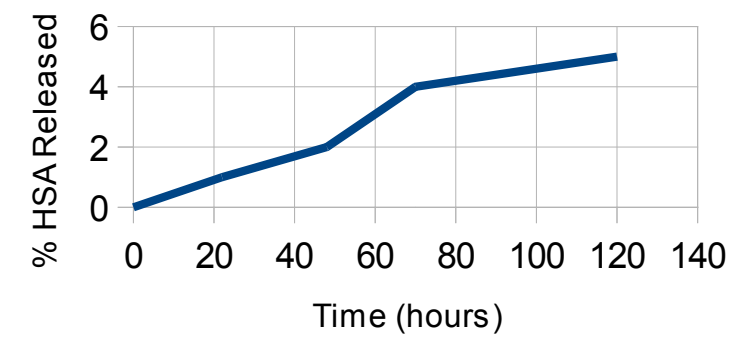

Figure 3. In vitro release of HSA from HSA-SPIONs loaded PLGA nanoparticles. 


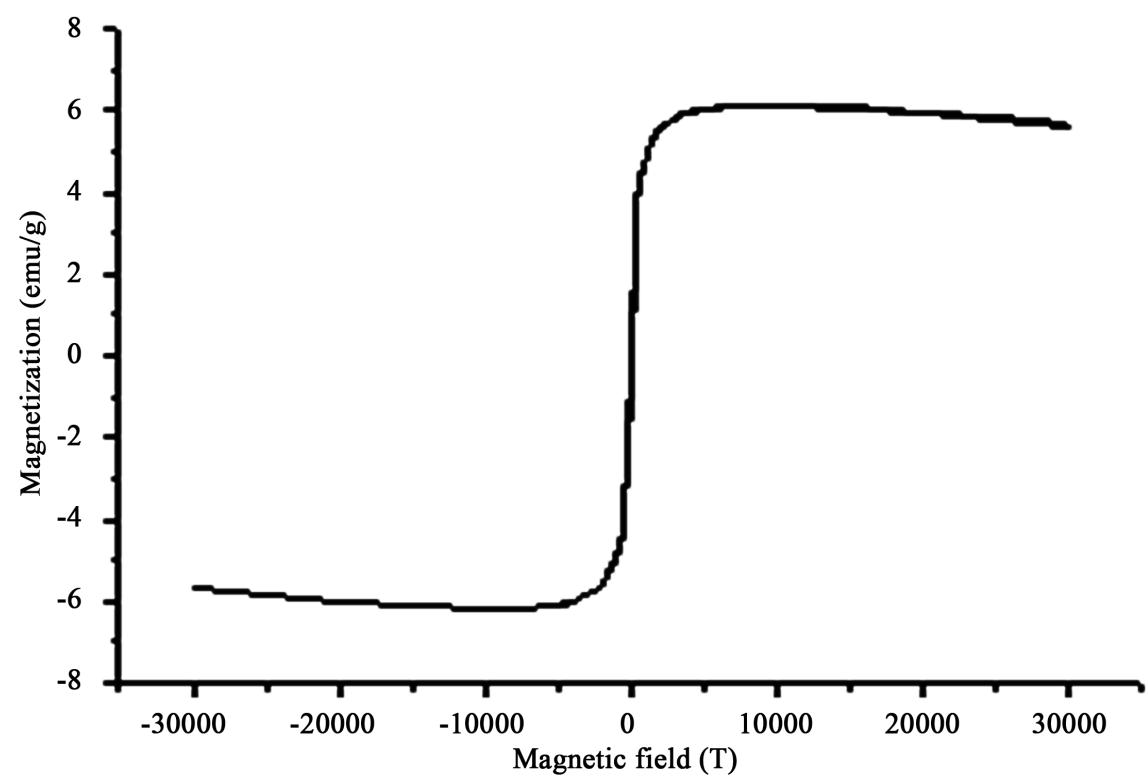

Figure 4. Hysteresis loop of HSA-SPIONs loaded PLGA nanoparticles.

\section{Conclusion}

Many important parameters are required in the synthesis of HSA-SPIONs loaded PLGA nanoparticles. In summarizing our data, we argued that HSA-SPIONS loaded PLGA nanoparticles are long term stability. These results have important indication for their potential applications such as in protein delivery.

\section{Acknowledgements}

This study was supported by the Erasmus Mundus II EXPERTS III (SV).

\section{Conflicts of Interest}

The authors declare no conflicts of interest regarding the publication of this paper.

\section{References}

[1] Lai, F., et al., (2006) Artemisia arborescens L. Essential Oil Loaded, Solid Lipid Nanoparticles for Potential Agricultural Application: Preparation and Characterization AAPS PharmSciTech, 7, E10. https://doi.org/10.1208/pt070102

[2] Huang, D., et al. (2003) Plastic Compatible Low Resistance Printable Gold Nanoparticle Conductors for Flexible Electronics. Journal of the Electrochemical Society, 150, G412-G417. https://doi.org/10.1149/1.1582466

[3] Choi, M.J., et al. (2008) Metal-Containing Nanoparticles and Nano-Structured Particles in Fingermark Detection. Forensic Science International, 179, 87-97. https://doi.org/10.1016/j.forsciint.2008.04.027

[4] Liu, T.M., et al. (2007) Nanoparticle Electric Propulsion for Space Exploration in Space Technology and Applications International Forum, STAIF.

[5] Jahanshahi, M. and Babaei, Z. (2008) Protein Nanoparticle: A Unique System as Drug Delivery Vehicles. African Journal of Biotechnology, 7, 4926. 
[6] Kawashima, Y., et al. (2000) Mucoadhesive DL-Lactide/Glycolide Copolymer Nanospheres Coated with Chitosan to Improve oral Delivery of Elcatonin. Pharmaceutical Development and Technology, 5, 77-85.

https://doi.org/10.1081/PDT-100100522

[7] Rieux, A.D., et al. (2006) Nanoparticles as Potential Oral Delivery Systems of Proteins and Vaccines: A Mechanistic Approach. Journal of Controlled Release, 116, 1-27. https://doi.org/10.1016/j.jconrel.2006.08.013

[8] Curtis, A.S.G. and Wilkinson, C. (2001) Nanotechniques and Approaches in Biotechnology. Trends in Biotechnology, 19, 97-101.

https://doi.org/10.1016/S0167-7799(00)01536-5

[9] Wilkinson, J.M. (2003) Nanotechnology Applications in Medicine. Medical Device Technology, 14, 29-31.

[10] Panyam, J. and Labhasetwar, V. (2003) Biodegradable Nanoparticles for Drug and Gene Delivery to Cells and Tissue. Advanced Drug Delivery Reviews, 55, 29-47. https://doi.org/10.1016/S0169-409X(02)00228-4

[11] Moghimi, S.M., Hunter, A.C. and Murray, J.C. (2001) Long-Circulating and Target Specific Nanoparticles: Theory to Practice. Pharmacological Reviews, 53, 283-318.

[12] Nicolas, J., Mura, S., Brambilla, D., Mackiewicz, N. and Couvreur, P. (2013) Design, Functionalization Strategies and Biomedical Applications of Targeted Biodegradable/Biocompatible Polymer-Based Nanocarriers for Drug Delivery. Chemical Society Reviews, 42, 1147-1235. https://doi.org/10.1039/C2CS35265F

[13] Soussan, E., Cassel, S., Blanzat, M. and Rico-Lattes, I. (2009) Drug Delivery by Soft Matter: Matrix and Vesicular Carriers. Angewandte Chemie International Edition, 48, 274-288. https://doi.org/10.1002/anie.200802453

[14] Brannon-Peppas, L. and Blanchette, J.O. (2004) Nanoparticle and Targeted Systems for Cancer Therapy. Advanced Drug Delivery Reviews, 56, 1649-1659. https://doi.org/10.1016/j.addr.2004.02.014

[15] Panyam, J. and Labhasetwar, V. (2003) Biodegradable Nanoparticles for Drug and Gene Delivery to Cells and Tissue. Advanced Drug Delivery Reviews, 55, 329-347. https://doi.org/10.1016/S0169-409X(02)00228-4

[16] Topete, A., Melgar, D., Alatorre-Meda, M., Iglesias, P., Argibay, B., Vidawati, S., Barbosa, S., Costoya, J.A., Taboada, P. and Mosquera, V. (2014) NIR Light Active Hybrid Nanoparticles for Combined Imaging and Bimodal Therapy of Cancerous Cells. Journal of Materials Chemistry B, 2, 6967-6977. https://doi.org/10.1039/C4TB01273A

[17] Prasad, S., Cody, V., Saucier-Sawyer, J.K., Saltzman, W.M., Sasaki, C.T., Edelson, R.L., Birchall, M.A. and Hanlon, D.J. (2011) Polymer Nanoparticles Containing Tumor Lysates as Antigen Delivery Vehicles for Dendritic Cell-Based Antitumor Immunotherapy. Nanomedicine, 7, 1-10. https://doi.org/10.1016/j.nano.2010.07.002

[18] Solbrig, C.M., Saucier-Sawyer, J.K., Cody, V., Saltzman, W.M. and Hanlon, D.J. (2007) Polymer Nanoparticles for Immunotherapy from Encapsulated $\mathrm{Tu}$ mor-Associated Antigens and Whole Tumor Cells. Molecular Pharmaceutics, 4, 47-57. https://doi.org/10.1021/mp060107e

[19] Thomas, C., Rawat, A., Hope-Weeks, L. and Ahsan, F. (2010) Aerosolized PLA and PLGA Nanoparticles Enhance Humoral, Mucosal and Cytokine Responses to Hepatitis B Vaccine. Molecular Pharmaceutics, 8, 405-415. https://doi.org/10.1021/mp100255c

[20] Arbab, A.S., Bashaw, L.A., Miller, B.R., Jordan, E.K., Lewis, B.K., Kalish, H. and Frank, J.A. (2003) Characterization of Biophysical and Metabolic Properties of Cells 
Labeled with Superparamagnetic Iron Oxide Nanoparticles and Transfection Agent for Cellular MR Imaging. Radiology, 229, 838-846.

https://doi.org/10.1148/radiol.2293021215

[21] Pankhurst, Q.A., Connolly, J., Jones, S.K. and Dobson, J. (2003) Applications of Magnetic Nanoparticles in Biomedicine. Journal of Physics D: Applied Physics, 36, R167-R181. https://doi.org/10.1088/0022-3727/36/13/201

[22] Wartlick, H., Michaelis, K., Balthasar, S., Strebhardt, K., Kreuter, J. and Langer, K. (2004) Highly Specific HER2-Mediated Cellular Uptake of Antibody-Modified Nanoparticles in Tumor Cells. Journal of Drug Targeting, 12, 461-471. https://doi.org/10.1080/10611860400010697

[23] Elzoghby, A.O., Samy, W.M. and Elgindy, N.A. (2012) Albumin-Based Nanoparticles as Potential Controlled Release Drug Delivery Systems. Journal of Controlled Release, 157, 168-182. https://doi.org/10.1016/j.jconrel.2011.07.031

[24] Kratz, F. (2008) Albumin as a Drug Carrier: Design of Prodrugs, Drug Conjugates and Nanoparticles. Journal of Controlled Release, 132, 171-183.

https://doi.org/10.1016/j.jconrel.2008.05.010

[25] Fasano, M., Curry, S., Terreno, E., Galliano, M., Fanali, G., Narciso, P., Notari, S. and Ascenzi, P. (2005) The Extraordinary Ligand Binding Properties of Human Serum Albumin. IUBMB Life, 57, 787-796. https://doi.org/10.1080/15216540500404093

[26] Can, K., Ozmen, M. and Ersoz, M. (2009) Immobilization of Albumin on Aminosilane Modified Superparamagnetic Magnetite Nanoparticles and Its Characterization. Colloids and Surfaces B: Biointerfaces, 71, 154-159.

https://doi.org/10.1016/j.colsurfb.2009.01.021

[27] van de Weert, M., Hennink, W.E. and Jiskoot, W. (2000) Protein Instability in Poly(Lactic-Coglycolic Acid) Microparticles. Pharmaceutical Research, 17, 1159-1167. https://doi.org/10.1023/A:1026498209874

[28] Steinmann, U., Braun, R., Sippel, H. and Estler, C.J. (1990) Effects of Naftidrofuryl on the Indocyanine Green Clearance and Cardiovascular Function of Rats with Liver Cirrhosis. Arzneimittelforschung, 40, 709-711. 